$3^{-p}$, either $F(n h)=0$ or $\Phi_{h}(n h)=0$. Thus the sum on the right-hand side of equation (3) is identically zero. Combining equation (3) with equation (2), we have the desired result.

* The research for this paper was supported by the United States Air Force under Contract No. AF18(600-685) monitored by the Office of Scientific Research.

${ }^{1}$ A discussion of the problem along with the necessary references will be found in Harry Pollard, "The Harmonic Analysis of Bounded Functions," Duke Math. J., 20, 499-512, 1953.

${ }^{2}$ See ibid.

${ }^{3}$ See S. Bochner, Fouriersche Integrale (Leipzig, 1932), p. 33.

4 The Levitan polynomial. See N.I. Achieser, Approximationstheorie (Berlin, 1953), p. 146.

\title{
A CENTRAL LIMIT THEOREM AND A STRONG MIXING CONDITION
}

\section{By M. Rosenblatt}

COLUMBIA UNIVERSITY

Communicated by P. A. Smith, November 10, 1955

Introduction.-This paper presents a central limit theorem for a sequence of dependent random variables

$$
X_{1}, X_{2}, \ldots \text {. }
$$

The assumptions required are the usual assumptions on second and $2+\delta$ order moments and a strong mixing condition. The theorem is of interest for two reasons. All general central limit theorems for dependent random variables formalize in some sense a heuristic notion of $\mathrm{A}$. Markoff to the effect that one expects a central limit theorem to hold for $X_{1}, X_{2}, \ldots$, if the random variables behave more like independent random variables the farther they are separated (assuming that appropriate moments exist). An interesting discussion of this intuitive notion is given in S. Bernstein's paper on the central limit theorem. ${ }^{1}$ The strong mixing condition used in this paper seems to be a more intuitively appealing formalization of this notion than most others. The condition is also of interest because it is a strong version of the mixing condition encountered in ergodic theory (see Hopf, ${ }^{2}$ p. 35).

The Mixing Condition.-The mixing condition is assumed to hold for sets of the form

$$
a_{k r}<X_{k r} \leq b_{k r}, \quad r=1, \ldots, s,
$$

where $k_{1}<\ldots<k_{s}$. Given any two such sets $A$ and $B$, introduce the following notion of distance $d(A, B)$ between them. Consider the smallest interval containing the indices of the random variables in terms of which $A$ is defined. Call this interval $I(A)$. In the case of set (1), this interval is $\left[k_{1}, k_{s}\right]$. The distance $d(A, B)$ is set equal to the distance between the intervals $I(A)$ and $I(B)$. Note that $d(A, B)$ does not satisfy the usual properties of a distance; for example, one may have $d(A$, $B)>d(A, C)+d(B, C)$ for some set $C$. The strong mixing condition is satisfied by the sequence of random variables $X_{1}, X_{2}, \ldots$, if 


$$
|P(A \cap B)-P(A) P(B)|<f(d(A, B))
$$

for all sets $A, B$ of form (1), where $f(n)$ is a function defined for $n=0,1,2, \ldots$. and decreasing to zero as $n \rightarrow \infty$.

The Central Limit Theorem.-Assume that the mean values $E X_{k} \equiv 0$, as one could in any case subtract the mean values $E X_{k}$ from the random variables $X_{k}$, respectively. The assumptions are

1. $E\left|\sum_{j=a}^{b} X_{j}\right|^{2} \sim h(b-a)$

as $b-a \rightarrow \infty$, where $h(m) \uparrow \infty$ as $m \rightarrow \infty$;

$$
E\left|\sum_{j=a}^{b} X_{j}\right|^{2+\delta}=o\left(h(b-a)^{1+\delta / 2}\right.
$$

as $b-a \rightarrow \infty$ for some $\delta>0$.

2. The strong mixing condition (2) is satisfied by the sequence of random variables $X_{1}, X_{2}, \ldots$.

Introduce the following notation:

$$
\begin{aligned}
& S_{n}=\sum_{j=1}^{n} X_{j} \\
& U_{r}=\sum_{j=(r-1)\left(p_{n}+q_{n}\right)+1}^{r p_{n}+(r-1) q_{n}} X_{j}, \\
& V_{r}=\sum_{j=r p_{n}+\left(p^{\prime}-1\right) q_{n}+1}^{r\left(p_{n}+q_{n}\right)} X_{j}, \quad r=1, \ldots, k,
\end{aligned}
$$

where $k\left(p_{n}+q_{n}\right)=n$. The numbers $k, p_{n}, q_{n}$ will be chosen in such a way that $p_{n}, q_{n}, k \rightarrow \infty$ and $q_{n} / p_{n} \rightarrow 0$ as $n \rightarrow \infty$.

We expect to show that

$$
\frac{S_{n}}{\sqrt{k h\left(p_{n}\right)}}
$$

is asymptotically normally distributed. Now

$$
\begin{gathered}
E^{1 / 2}\left|\sum_{r=1}^{k} \frac{V_{r}}{\sqrt{k h\left(p_{n}\right)}}\right|^{2} \leq \sum_{r=1}^{k} \frac{E^{1 / 2}\left|V_{r}\right|^{2}}{\sqrt{k h\left(p_{n}\right)}} \\
\sim \frac{\left(k h\left(q_{n}\right)\right.}{\left.h\left(p_{n}\right)\right)^{1 / 2} .}
\end{gathered}
$$

Choose the sequences $q_{n}, p_{n}, k$ such that

$$
\frac{k h\left(q_{n}\right)}{h\left(p_{n}\right)} \rightarrow 0 .
$$

Then

$$
\sum_{r=1}^{k} \frac{V_{r}}{\sqrt{k h\left(p_{n}\right)}} \rightarrow 0
$$


in probability as $n \rightarrow \infty$. It can therefore be neglected in further computations. We shall later see that this can be done.

Set

$$
G_{r, n}(x)=P\left\{\frac{U_{r}}{\sqrt{k h\left(p_{n}\right)}} \leq x\right\}
$$

and let the set

$$
\left\{m_{r} \delta<\frac{U_{r}}{\sqrt{k h\left(p_{n}\right)}} \leq\left(m_{r}+1\right) \delta\right\}=A\left(r, n, m_{r}, \delta\right),
$$

where $m_{r}$ is an integer. Note that

$$
\begin{array}{r}
\sum_{\left(m_{1}+\ldots+m_{k}+k\right) \leq x} P\left(\bigcap_{r=1}^{k} A\left(r, n, m_{r} ; \delta\right)\right) \leq P\left(\sum_{r=1}^{k} \frac{U_{r}}{\sqrt{k h\left(p_{n}\right)}} \leq x\right) \leq \quad(3) \\
\sum_{\left(m_{1}+\ldots+m_{k}\right) \delta \leq x} P\left(\bigcap_{r=1}^{k} A\left(r, n, m_{r}, \delta\right)\right)
\end{array}
$$

Lemma 1

$$
P\left(\max _{r=1, \ldots, k}\left|\frac{U_{r}}{\sqrt{k h\left(p_{n}\right)}}\right|>t_{k}\right)<\epsilon
$$

if $t_{k}=(k / \epsilon)^{1 / 2}$.

Now

$$
E\left|\max _{r=1, \ldots, k}\right| \frac{U_{r}}{\sqrt{k h\left(p_{n}\right)}}||^{2} \leq E\left|\sum_{r=1}^{k}\right| \frac{U_{r}}{\sqrt{k h\left(p_{n}\right)}}||^{2} \leq k .
$$

The result is obtained by an application of the Tchbycheff inequality.

Lemma 2

$$
\begin{gathered}
\left|\sum_{\left(m_{1}+\ldots+m_{k}\right) \delta \leq x} P\left(\bigcap_{r=1}^{k} A\left(r, n, m_{r}, \delta\right)\right)-\sum_{\left(m_{1}+\ldots+m_{k}\right) \delta \leq x} \prod_{r=1} P\left(A\left(r, n, m_{r}, \delta\right)\right)\right| \\
\leq k\left(\frac{2 t_{k}}{\delta}\right)^{k} f\left(q_{n}\right)+2 \epsilon .
\end{gathered}
$$

The probability contributed by all the sets $\bigcap_{r=1}^{k} A\left(r, n, m_{r}, \delta\right)$ for which max $\left|U_{r} / \sqrt{k h\left(p_{n}\right)}\right|>t_{k}$ is at most $\epsilon$. Consider the sets $\bigcap_{r=1}^{k} A\left(r, n, m_{r}, \delta\right)$ for which $\max \left|U_{r} / \sqrt{k h\left(p_{n}\right)}\right| \leq t_{k}$. By repeated application of condition (2), one can see that

$$
\left|P\left(\bigcap_{r=1}^{k} A\left(r, n, m_{r}, \delta\right)\right)-\prod_{r=1}^{k} P\left(A\left(r, n, m_{\tau}, \delta\right)\right)\right| \leq k f\left(q_{n}\right) .
$$

Since there are $\left(2 t_{k} / \delta\right)^{k}$ sets of this form, we obtain the desired inequality.

Let

$$
G_{1, n} * \ldots * G_{k, n}(x)
$$


be the convolution of $G_{1, n}(x), \ldots, G_{k, n}(x)$. Note that

$$
\begin{array}{r}
G_{1, n} * \ldots * G_{k, n}(x) \leq \sum_{\left(m_{1}+\ldots+m_{k}\right) \delta \leq x} \prod_{r=1}^{k} P\left(A\left(r, n, m_{\tau}, \delta\right)\right) \\
\leq G_{1, n} * \ldots * G_{k, n}(x+k \delta)
\end{array}
$$

and

$$
\begin{array}{r}
G_{1, n} * \ldots * G_{k, n}(x-k \delta)<\sum_{\left(m_{1}+\ldots+m_{k}+k\right) \delta \leq x} \prod_{r=1}^{k} P\left(A\left(r, n, m_{r}, \delta\right)\right) \\
\leq G_{1, n} * \ldots * G_{k, n}(x) .
\end{array}
$$

Now $G_{1, n} * \ldots * G_{k, n}(x)$ tends to the normal distribution as $n \rightarrow \infty$ if $k \rightarrow \infty$. We want to let $k, p_{n}, q_{n} \rightarrow \infty, k p_{n} \sim n$, and $k \delta \rightarrow 0$ in such a way that

$$
\begin{gathered}
k\left(\frac{2 t_{k}}{\delta}\right)^{k} f\left(q_{n}\right) \rightarrow 0 \\
\frac{k h\left(q_{n}\right)}{h\left(p_{n}\right)} \rightarrow 0 .
\end{gathered}
$$

Set $\delta=1 / k^{2}$. Then $k \delta \rightarrow 0$. Since

$$
t_{k}=\left(\frac{k}{\epsilon}\right)^{1 / 2}
$$

it follows that

$$
k\left(\frac{2 t_{k}}{\delta}\right)^{k}=k^{5 / 2 k+1} \frac{2^{k}}{\epsilon^{1 / 2 k}} \leq k^{5 k} C^{k},
$$

where $C=2 / \epsilon^{1 / 2}$. The function $f$ can always be chosen in such a way that $f(n)>$ $1 / n$ for all $n$. If $k$ is chosen so that

$$
k \leq\left[-\log f\left(q_{n}\right)\right]^{1 / 2},
$$

clearly condition (6) will be satisfied.

Now

$$
h(n) \sim E\left|\sum_{j=1}^{n} X_{j}\right|^{2} \leq k^{2} h\left(\frac{n}{k}\right)
$$

by the Minkowski inequality. But then

$$
\frac{k^{2}}{h(n)} \geq \frac{1}{h\left(p_{n}\right)}
$$

Thus

$$
\frac{k h\left(q_{n}\right)}{h\left(p_{n}\right)} \leq \frac{k^{3} h\left(q_{n}\right)}{h(n)} .
$$

Condition (4) will then be satisfied if

$$
h\left(q_{n}\right)=o\left(\frac{h(n)}{k^{3}}\right) .
$$


Sequences $k, p_{n}, q_{n}, \rightarrow \infty, k p_{n} \sim n$ can be chosen so that conditions (8) and (9) are satisfied and hence conditions (6) and (7) are satisfied. On using inequalities (3). (4), and (5) and Lemma 2 , we see that

$$
\sum_{r=1}^{k} \frac{U_{r}}{\sqrt{k h\left(p_{n}\right)}}
$$

is asymptotically normally distributed. Since

$$
\sum_{r=1}^{k} \frac{V_{r}}{\sqrt{k h\left(p_{n}\right)}} \rightarrow 0
$$

in probability as $n \rightarrow \infty$, it follows that

$$
\frac{S_{n}}{\sqrt{k h\left(p_{n}\right)}}
$$

is asymptotically normally distributed.

Remarks.-The result obtained includes the result of Höffding and Robbins. ${ }^{3}$ It would be of very great interest to see how much stronger the notion of a strong mixing condition is than that of an ordinary mixing condition in the case of a strictly stationary process.

${ }^{1} \mathrm{~S}$. Bernstein, "Sur l'extension du théorème limite du calcul des probabilités aux sommes de quantités dépendentes," Math. Ann., 97, 1-59, 1927.

${ }^{2}$ E. Hopf, Ergodentheorie ("Ergebnisse" series). J. Springer, Berlin, 1937.

${ }^{3}$ W. Höffding and H. Robbins, "The Central Limit Theorem for Dependent Random Variables," Duke Math. J., 15, 773-780, 1948. 